Article

\title{
Development of a Protein Scaffold for Arginine Sensing Generated through the Dissection of the Arginine-Binding Protein from Thermotoga maritima
}

\author{
Giovanni Smaldone ${ }^{1, *(\mathbb{C})}$, Alessia Ruggiero ${ }^{2, *}$, Nicole Balasco ${ }^{2}\left[\right.$ and Luigi Vitagliano ${ }^{2} \mathbb{C}$ \\ 1 IRCCS SDN, Via Emanuele Gianturco, 11380143 Naples, Italy \\ 2 Institute of Biostructures and Bioimaging, CNR, Via Mezzocannone 16. I-80134 Naples, Italy; \\ nicole.balasco@unina.it (N.B.); luigi.vitagliano@unina.it (L.V.) \\ * Correspondence: giovanni.smaldone@synlab.it (G.S.); alessia.ruggiero@unina.it (A.R.)
}

Received: 9 September 2020; Accepted: 8 October 2020; Published: 12 October 2020

\begin{abstract}
Arginine is one of the most important nutrients of living organisms as it plays a major role in important biological pathways. However, the accumulation of arginine as consequence of metabolic defects causes hyperargininemia, an autosomal recessive disorder. Therefore, the efficient detection of the arginine is a field of relevant biomedical/biotechnological interest. Here, we developed protein variants suitable for arginine sensing by mutating and dissecting the multimeric and multidomain structure of Thermotoga maritima arginine-binding protein (TmArgBP). Indeed, previous studies have shown that TmArgBP domain-swapped structure can be manipulated to generate simplified monomeric and single domain scaffolds. On both these stable scaffolds, to measure tryptophan fluorescence variations associated with the arginine binding, a Phe residue of the ligand binding pocket was mutated to Trp. Upon arginine binding, both mutants displayed a clear variation of the Trp fluorescence. Notably, the single domain scaffold variant exhibited a good affinity $(\sim 3 \mu \mathrm{M})$ for the ligand. Moreover, the arginine binding to this variant could be easily reverted under very mild conditions. Atomic-level data on the recognition process between the scaffold and the arginine were obtained through the determination of the crystal structure of the adduct. Collectively, present data indicate that $\mathrm{TmArgBP}$ scaffolds represent promising candidates for developing arginine biosensors.
\end{abstract}

Keywords: protein scaffolds; argininemia diagnosis; biosensors; crystal structure; protein dissection; protein stability

\section{Introduction}

L-arginine, thereafter denoted as arginine, is a fundamental nutrient of living organisms [1]. Indeed, this amino acid, which is semi-essential for mammals, is required in protein synthesis and in other important metabolic pathways. Arg is also a precursor for the synthesis of nitric oxide (NO), making it important in the regulation of blood pressure and in tumorigenesis [2-5]. However, arginine metabolism is complex, as multiple enzymes utilize this metabolite as substrate [6].

The accumulation of arginine in the human body as a consequence of defects in the arginase I enzyme causes hyperargininemia (OMIN 207800), an autosomal recessive disorder [7]. Alterations of enzymes linked to arginine metabolism have been recently implicated in Alzheimer disease (AD) [8].

In this scenario, the efficient detection and quantification of the arginine is a field of relevant biomedical/biotechnological interest. Natural arginine binders are the evident candidates for developing this type of biosensors. Among these, substrate-binding proteins (SBP) that deliver arginine and other metabolites to the $\mathrm{ABC}$ cassette system for their transportation across the periplasmatic membrane appear to be particularly appropriate for their specificity and stability. In the last few years, we 
have characterized the arginine-binding protein isolated from the hyperthermophilic organism Thermotoga maritima (TmArgBP) [9-13]. This protein presents a number of distinctive/unique features in the large class of SBP. Although substrate-binding proteins are generally monomeric, TmArgBP is essentially dimeric, with the concomitant presence, as minor components, of higher oligomerization states [14-16]. The crystallographic structure of the protein has highlighted that its dimer is stabilized by swapping of the terminal C-terminal helix [9] (Figure 1). The three-dimensional structure of the protein has also shown that the arginine binding does not only produce a remarkable tertiary structure variation of TmArgBP but also a radical rearrangement of its quaternary structure. The biophysical characterization of the protein has also revealed that it is endowed with an unusual stability against temperature, chemical denaturants and pressure [12,17-19]. We have exploited the extraordinary stability of TmArgBP to effectively manipulate the protein through mutation, truncation and dissection of its native structure $[10,13,14]$. In particular, we have shown that a stable monomeric form may be obtained either though site-directed mutagenesis [15] or through the deletion of the C-terminal end, the swapping element in the protein binding [10]. TmArgBP can be further manipulated by dissecting it into its constitutive D1 and D2 domains (Figure 1).

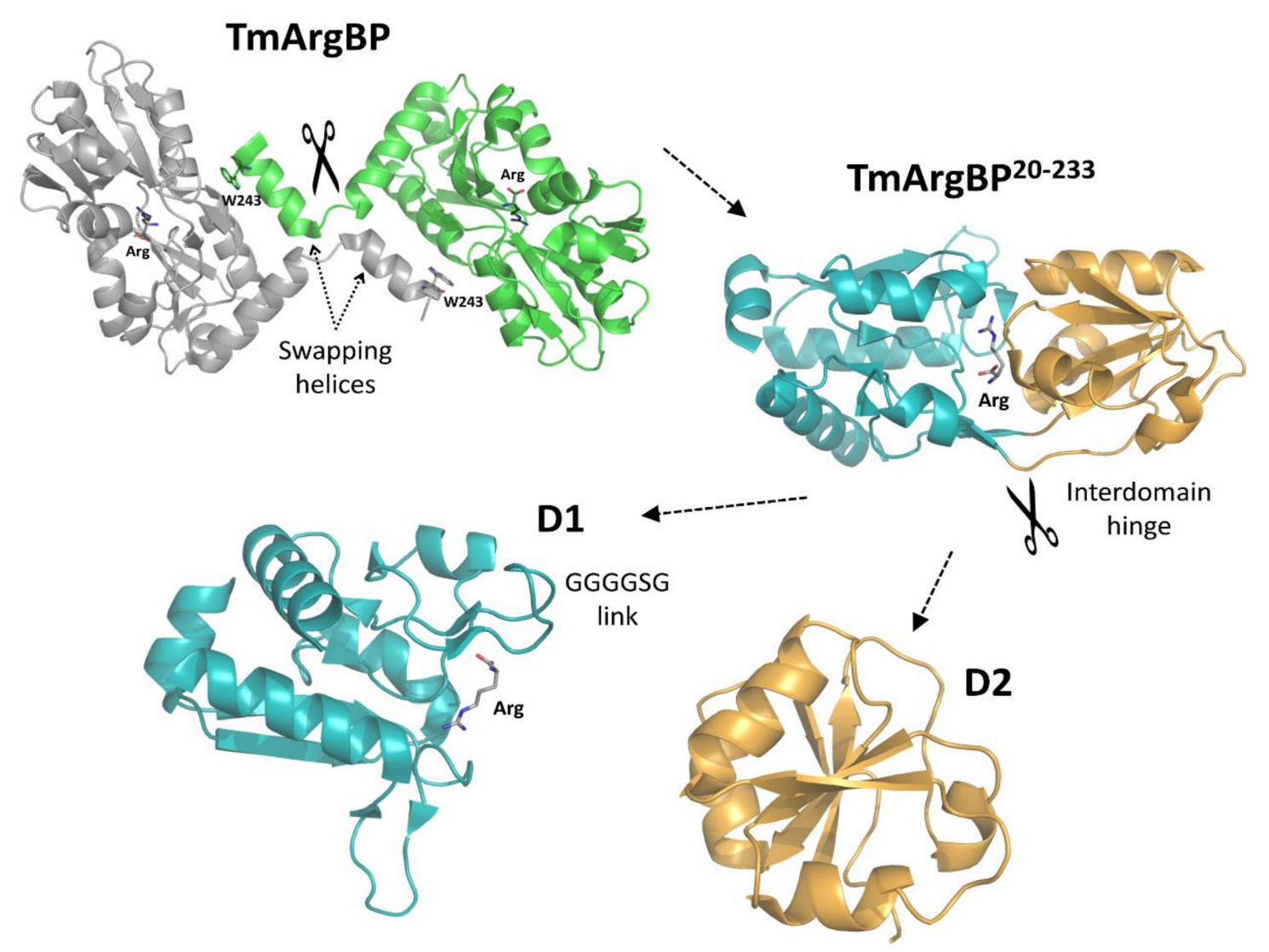

Figure 1. Cartoon representation of Thermotoga maritima arginine-binding protein (TmArgBP) truncation and dissection from the wild-type dimer to the truncated monomeric form TmArgBP $20-233$ and, finally, to the individual D1 and D2 domains. The arginine-bound form of TmArgBP (ProteinDataBank ID: 4PSH), of TmArgBP20-233 (PDB ID: 6GGV), of D1 (PDB ID: 6GPC) and the D2 domain (PDB ID: 6GPM) have been used to generate the figure. The arginine ligand and residue Trp243 are shown as sticks.

Taking into account these properties, TmArgBP appears a promising system for developing an arginine biosensor. Unfortunately, no fluorescence signal is emitted by the protein due to the lack of Trp residues close to the arginine-binding site. Indeed, the only Trp present in the sequence of the protein (W243) is located at the C-terminal end of the protein far from the arginine binding 
pocket (Figure 1). Therefore, any attempt to devise a TmArgBP-based sensor must proceed through modifications of the wild-type protein. Interesting variants of the protein have been hitherto developed by using as a scaffold the TmArgBP dimer [16,20]. Taking into account the relative inhomogeneity of the wild-type TmArgBP that presents trimeric and higher oligomeric forms along with the dimeric state and the virtually irreversible arginine binding to the protein, we here attempted to generate novel TmArgBP-derived scaffolds using simplified variants. In particular, we here exploited the possibility to manipulate this protein to generate potential arginine sensors. Through combined dissections and mutations of TmArgBP, we were able to generate distinct protein scaffolds whose tryptophan fluorescence was sensitive to the arginine binding (Figure S1). In more general terms, our data also demonstrate that extremely stable substrate-binding proteins, which are very promising systems for developing biosensors, may be effectively manipulated to obtain simplified scaffolds that also reversibly bind the ligand.

\section{Results}

\subsection{Design of a Potentially Fluorescent Mutant and its Characterization by Computational Techniques}

The inspection of the binding pocket of TmArgBP indicates that the protein anchors the aminoacid through a network of different types of interactions. These include hydrophobic interactions involving both aliphatic and aromatic residues, hydrogen bonds and salt bridges that are deputed to the binding of the three charged terminal groups of arginine. Of particular relevance is the interaction that the guanidinium moiety of the ligand makes with the side chains of Phe38 and Phe76 (Figure S2) [21]. Based on these observations, we identified Phe76 as a promising candidate to be replaced with a Trp residue since its environment is highly depending on the binding state of the protein. To this aim, by using computational techniques, we preliminary investigated the impact of a Phe $\rightarrow$ Trp mutagenesis on this site. In principle, the replacement of the Phe side chain with the bulkier indole group of Trp could induce some local clashes that could limit or even prevent the binding of the ligand. Since we were interested in designing simplified $\operatorname{TmArgBP}$ variants, we used the structure of the truncated monomeric variant TmArgBP ${ }^{20-233}$ as a scaffold. Initially, we manually modelled the Trp side chain inside the binding pocket by exploring the most frequent rotameric states of the residue [22]. Although most of these rotamers could not be allocated into the binding pocket without major steric clashes, the one characterized by $\chi^{1}$ and $\chi^{2}$ dihedral angles of $178^{\circ}$ and $105^{\circ}$ did not produce any local strain within the protein and presumably did not hamper the arginine binding (Figure S3).

In order to corroborate this preliminary observation, we performed a molecular dynamics (MD) simulation on the $\mathrm{F} 76 \mathrm{~W}$ variant of $\operatorname{TmArgBP}{ }^{20-233}\left(\operatorname{TmArgBP}{ }^{20-233}{ }^{\mathrm{F} 76 \mathrm{~W}}\right)$ in its arginine-bound state. The analysis of the structural parameters that are commonly used to evaluate the stability of trajectory structures indicates that $\mathrm{TmArg} \mathrm{B}^{\mathrm{P} 20-233-\mathrm{F} 76 \mathrm{~W}}$ evolves to reach rather stable states in the time interval 20-150 ns (Figure S4). The analysis of the mobility of the W76 throughout the simulation indicates that its side chain assumes a single rotameric state characterized by trans- and gauche- states for $\chi 1$ and $\chi 2$, respectively (Figure 2A). The visual inspection of the trajectory structures also shows that the arginine is located in the pocket (Figure 2). The analysis of the interactions that the ligand establishes with the protein indicates that it forms stable H-bonds, salt bridges (Figure S5), and hydrophobic interactions (Figure 2E and Figure S6). As shown in Figure 2B,C, the ligand is in close contact with the residue W76 whose environment is expected to change as a function of the protein-binding state. Collectively, these analyses suggest that the mutant $\mathrm{TmArgBP}{ }^{20-233-} \mathrm{F} 76 \mathrm{~W}$ is a suitable candidate to generate a protein variant that exhibits a variation of the Trp fluorescence as a consequence of the arginine binding. 


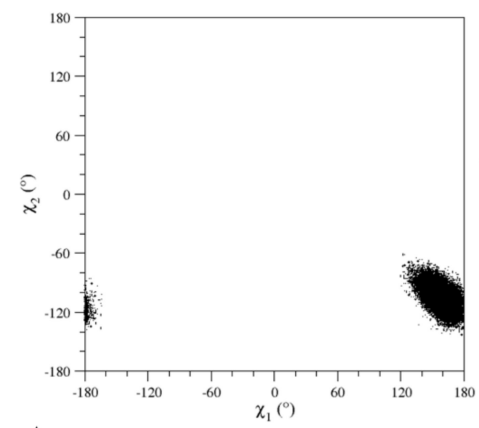

A

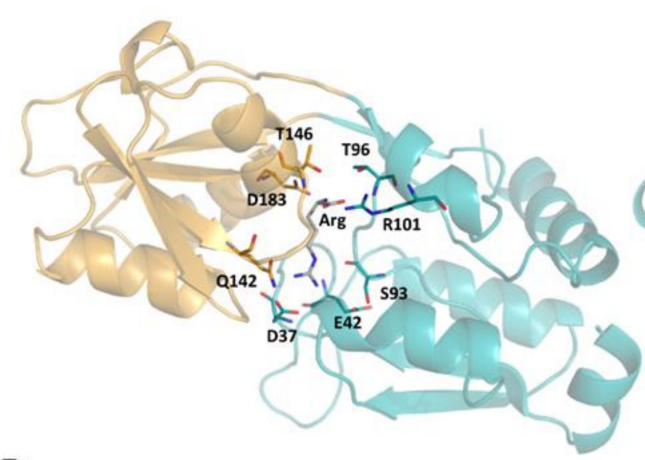

D
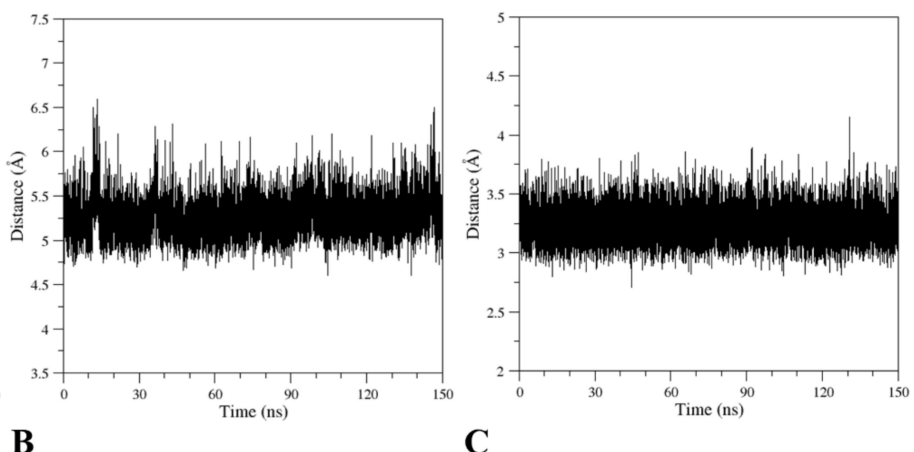

C

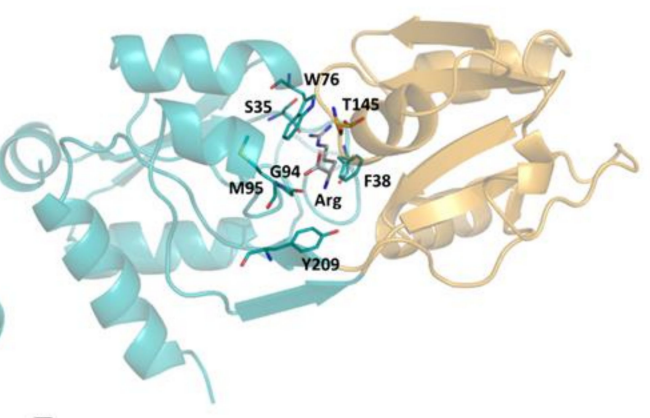

$\mathbf{E}$

Figure 2. Rotameric states ( $\chi^{1}$ and $\chi^{2}$ dihedral angles) assumed by Trp76 in the molecular dynamics (MD) simulation of TmArgBP20-233_F76W (A). Time evolution of the distances between: (B) the centers of mass and $(\mathbf{C})$ the closest atoms of the arginine ligand and Trp76 residue. Cartoon representation of the arginine environment: residues involved in H-bonding (D) or hydrophobic (E) interactions with the ligand are shown as sticks. The MD structure $(t=77.66 \mathrm{~ns})$ closest to the average structure computed in the equilibrated region of the trajectory (20-150 ns) has been considered.

\subsection{Expression and Characterization of the Mutant TmArgBP20-233_F76W}

To generate candidates for the development of an arginine biosensor based on simplified variants of TmArgBP, we expressed and characterized the mutant $\operatorname{TmArgBP}{ }^{20-233}{ }_{-} \mathrm{F} 76 \mathrm{~W}$, in which the mutation is inserted in the truncated variant of the protein (see Figure S1 for the sequence). The recombinant protein was expressed in high yields and remarkable purity. As expected on the basis of the data available for the parent protein and taking into account the deletion of the swapping element, the mutant is monomeric.

The folding state and the stability of TmArgBP $20-233 \_F 76 \mathrm{~W}$ was evaluated by circular dichroism (CD) spectroscopy. As shown in Figure 3A, the spectrum of the mutant displays the characteristic features shared by proteins adopting an $\alpha / \beta$ fold. It is also endowed with a remarkable stability as the thermal denaturation curve registered following the $C D$ signal at $222 \mathrm{~nm}$ does not present any significant transition (Figure 3B). This observation indicates that, as the parent protein [10], $\operatorname{Tm} A r g B P^{20-233}-\mathrm{F} 76 \mathrm{~W}$ is stable over the entire range of temperature explored $\left(20-100{ }^{\circ} \mathrm{C}\right)$. To induce the unfolding of this mutant, the increase in the temperature has to be coupled with the addition of $4.0 \mathrm{M}$ $\mathrm{GuHCl}$ (Figure 3C). In these conditions, the observed melting temperature is $57^{\circ} \mathrm{C}$.

Since TmArgBP and its variants are produced in arginine-bound forms in the commonly used expression protocols, the ligand was removed using rather harsh conditions that included the presence of the chemical denaturant $\mathrm{GuHCl}$ (see Methods for details). The spectrum corresponding to the Trp-fluorescence emission of the unliganded form of TmArgBP ${ }^{20-233-F 76 W}$ highlights the occurrence of a strong peak centered at $350 \mathrm{~nm}$ (Figure 4A). Since the deletion of the C-terminus of TmArgBP also eliminated the only tryptophan residue (W243) present in the wild-type protein, this emission is 
exclusively due to the tryptophan introduced in the active site upon mutation. To qualitatively and quantitatively measure the effect on the tryptophan fluorescence, $\operatorname{TmArgBP}{ }^{20-233-F 76 \mathrm{~W}}$ was titrated with increasing amounts of arginine (range 0.5-730 $\mu \mathrm{M}$ ). As shown in Figure 4A, the addition of the ligand produced significant effects on the fluorescence spectrum. Indeed, a remarkable reduction in the fluorescence intensity was observed along with a shift of the peak toward shorter wavelengths $(\sim 10 \mathrm{~nm})$ in a ligand concentration-dependent manner. Such a shift of fluorescence peaks, which are known to be generated from the tryptophan becoming more buried and shielded away from the hydrophilic environment, represents a strong indication of changes in the local environment of this residue [23]. This variation was quantified by plotting the peak of the tryptophan fluorescence in response to the ligand concentration (Figure 4B). The fitting of the experimental data also provides an estimate of the dissociation constant $\left(\mathrm{K}_{\mathrm{D}}=98.3 \pm 29 \mu \mathrm{M}\right)$ of the adduct formed by the arginine with $\mathrm{TmArgBP}{ }^{20-233 \_F 76 W}$. Finally, we evaluated the reversibility of the binding by washing the TmArgBP20-233_F76W/arginine complex with a Tris buffer solution and dialyzing it for four hours (see Section Materials and Methods for details). Then, the washed sample was titrated with arginine. As shown in Figure 4C, the addition of the ligand did not produce appreciable effects on the fluorescence spectrum. This indicates that the washing procedure adopted could not remove the tightly bound arginine from the protein pocket. This consideration is corroborated by the fact that the value of the wavelength $(\sim 335 \mathrm{~nm})$ of the intensity peak observed before the addition of the ligand (Figure $4 \mathrm{C})$ is similar to the one observed for the protein saturated with arginine (Figure 4A).

A

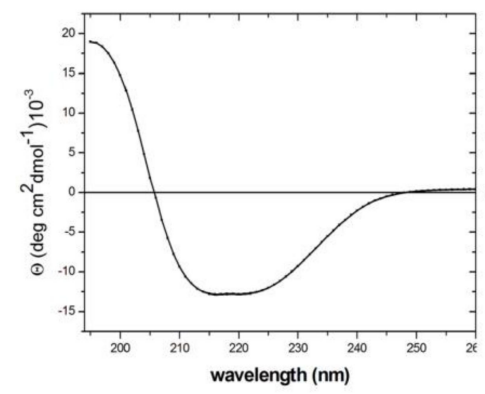

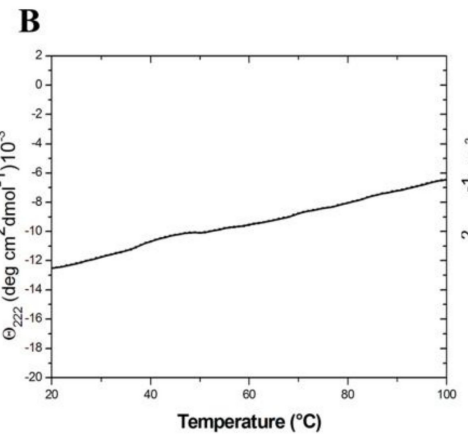

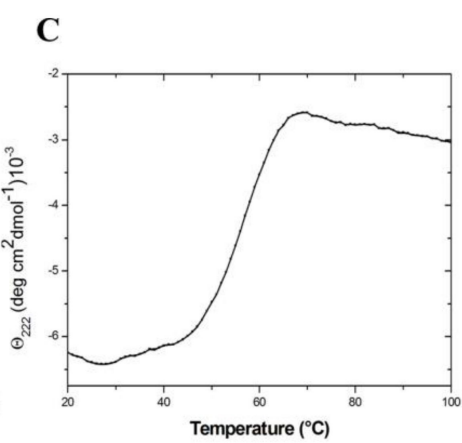

Figure 3. Spectroscopic characterization of TmArgBP $20-233$ F76W: Far-UV circular dichroism (CD) spectrum (A), thermal denaturation curves in phosphate buffer saline (PBS) buffer (B) and in PBS buffer containing 4.0 M GuHCl (C).

A

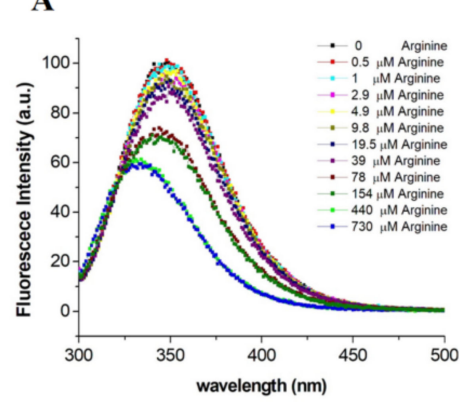

B

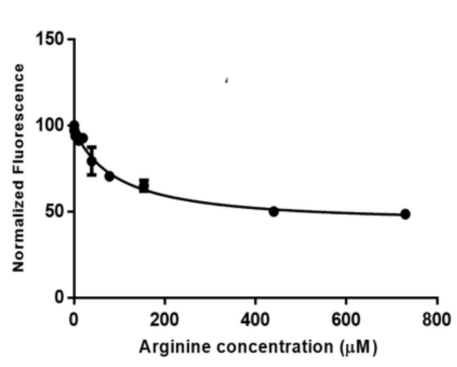

C

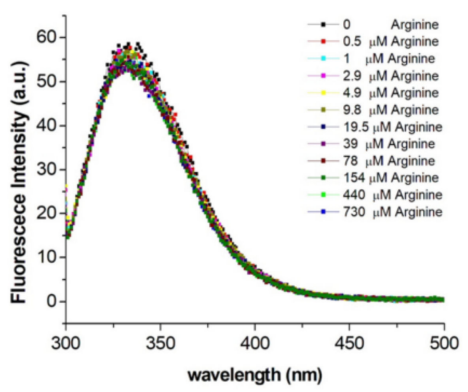

Figure 4. (A) Effect of the arginine binding on the Trp fluorescence of TmArgBP $20-233 \_F 76 \mathrm{~W}$. The concentration of the protein was $45 \mu \mathrm{M}$. (B) Maximum of the fluorescence intensity is reported as a function of the concentration. (C) Fluorescence measured on a TmArgBP ${ }^{20-233 \_F 76 W}$ sample that was initially saturated with arginine and then extensively washed. 


\subsection{From the Monomeric to a Single Domain Scaffold}

In our efforts to simplify the protein scaffold, we dissected the two-domain structure of the protein into its individual domains (D1 and D2). Although both domains cooperate to the anchoring of the arginine in the wild-type protein (Figure S1), only D1 is able to bind this ligand when considered as isolated entity. In this scenario, we introduced the same Phe $\rightarrow \operatorname{Trp}$ mutation we have previously characterized in TmArgBP ${ }^{20-233}$. Due to the different numbering scheme adopted for TmArgBP D1 (see the notations section in the Materials and Methods), the mutation is F57W and the mutant is denoted thereafter $\mathrm{D} 1^{\mathrm{F} 57 \mathrm{~W}}$. As for TmArgBP $20-233 \_\mathrm{F} 76 \mathrm{~W}$, a highly pure recombinant form of $\mathrm{D} 1^{\mathrm{F} 57 \mathrm{~W}}$ was expressed in high yields.

The $\mathrm{CD}$ spectrum indicates that $\mathrm{D} 1^{\mathrm{F} 57 \mathrm{~W}}$ assumes the expected $\alpha / \beta$ fold (Figure $5 \mathrm{~A}$ ). As for TmArgBP20-233_F76W , the thermal denaturation curve registered following the CD signal at $222 \mathrm{~nm}$ does not present any significant transition (Figure $5 \mathrm{~B}$ ). The melting point of $\mathrm{D} 1^{\mathrm{F} 57 \mathrm{~W}}$ was detected at $57^{\circ} \mathrm{C}$ when $4.0 \mathrm{M} \mathrm{GuHCl}$ was added to the protein solution (Figure $5 \mathrm{C}$ ).

A

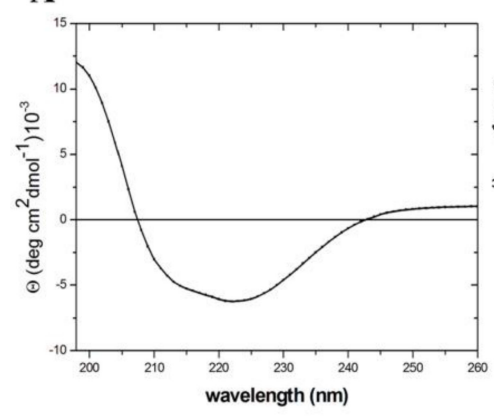

B

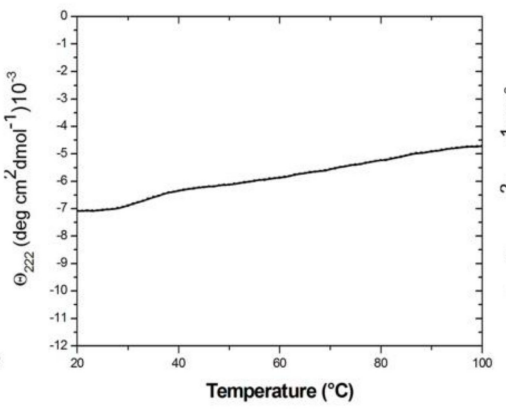

C

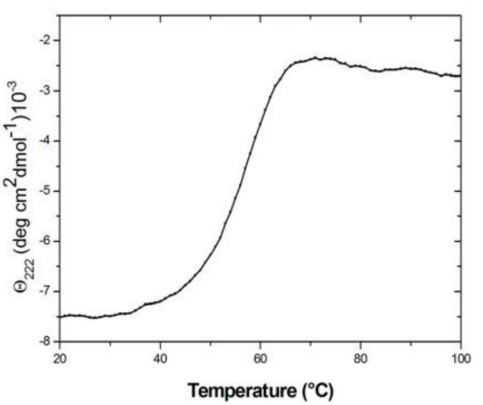

Figure 5. Spectroscopic characterization of $\mathrm{D} 1^{\mathrm{F57W}}$ : Far-UV CD spectrum (A), thermal denaturation curves in PBS buffer (B) and in PBS buffer containing 4.0 M GuHCl (C).

The tryptophan fluorescence spectrum of this mutant was very similar to that detected for TmArgBP $20-233 \_F 76 \mathrm{~W}$ with a peak of intensity centered at $350 \mathrm{~nm}$ (Figure 6A). The intensity and the wavelength of the peak change, in a dose-dependent manner, upon the addition of aliquots of arginine. The fitting of the intensity of the peak collected as function of the arginine concentration yields a $\mathrm{k}_{\mathrm{D}}$ value of $3.4 \pm 0.9 \mu \mathrm{M}$ (Figure $6 \mathrm{~B}$ ). It is worth mentioning that the dissociation constant observed for $\mathrm{D}^{\mathrm{F} 57 \mathrm{~W}}$ is significantly lower than that measured for $\operatorname{Tm} A \operatorname{ArgBP}{ }^{20-233}$. The analysis of the reversibility of the binding of arginine to $\mathrm{D} 1^{\mathrm{F} 57 \mathrm{~W}}$ provided quite different results than that obtained for TmArgBP $20-233-F 76 \mathrm{~W}$. Indeed, as shown in Figure 6C, the binding of the arginine to this protein scaffold is reversible, as the mutant was able again to bind the ligand after the application of the washing protocol also used for TmArgBP ${ }^{20-233 \_F 76 W}$ (see methods for details). Since we have recently shown that the D1 domain of TmArgBP is able to bind the guanidinium ion [17], we also checked whether $\mathrm{D} 1^{\mathrm{F} 57 \mathrm{~W}}$ was a suitable candidate for guanidinium sensing. However, as shown in Figure $6 \mathrm{D}$, the addition of this ion did not produce significant variations of the tryptophan fluorescence of the protein.

\subsection{Crystal Structure of $D 1^{F 57 W}$}

To gain further insights into the properties of the novel TmArgBP variants here designed and characterized here, we undertook crystallographic studies on $\mathrm{D} 1^{\mathrm{F} 57 \mathrm{~W}}$. The domain was successfully crystallized and diffraction data were collected at $1.79 \AA$ A resolution (Table 1). A three-dimensional model for $\mathrm{D} 1^{\mathrm{F} 57 \mathrm{~W}}$ was built on the basis of the electron density maps that were well-defined for the vast majority of the residues. The final model presents good crystallographic indicators and an excellent stereochemistry (Table 1 and Table S1 and Figure S7) that was evaluated using both standard and innovative protocols (see Methods). The asymmetric unit of the crystals contains two copies of the 
domain whose structure is very similar both at local and at global level (the RMSD value of the two copies is $0.31 \AA$ ).

A

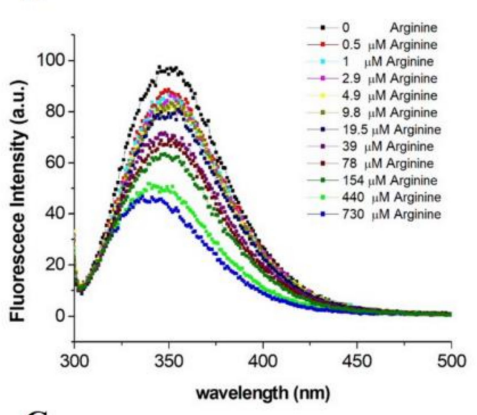

C

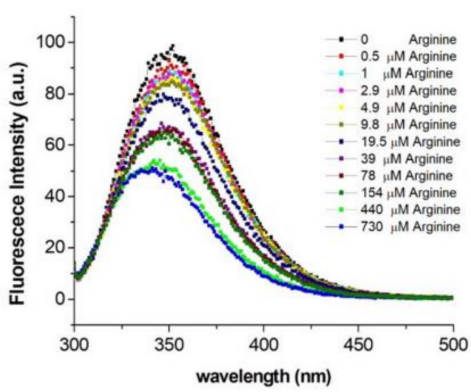

B

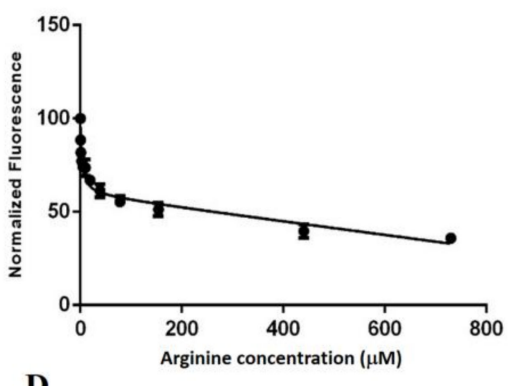

D

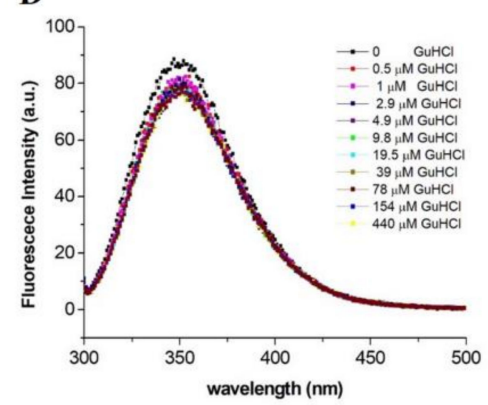

Figure 6. (A) Effect of the arginine binding on the Trp fluorescence of $\mathrm{D} 1^{\mathrm{F} 57 \mathrm{~W}}$. The concentration of the protein was $45 \mu \mathrm{M}$. (B) Maximum of the fluorescence intensity reported as function of the concentration. (C) Fluorescence measured on a $\mathrm{D} 1^{\mathrm{F} 57 \mathrm{~W}}$ sample that was initially saturated with arginine and then extensively washed. (D) Variation of the Trp fluorescence of $\mathrm{D1}^{\mathrm{F} 57 \mathrm{~W}}$ upon $\mathrm{GuHCl}$ titration.

Table 1. Data collection and refinement statistics of $\mathrm{TmArgBP} D 1^{\mathrm{F} 7 \mathrm{~W}}$. Values in parentheses are for the highest-resolution shell (1.87-1.79 ̊).

\begin{tabular}{cc}
\hline Protein & TmArgBP D1 ${ }^{\text {F57W }}$ \\
\hline X-ray device & Rigaku FR007HF with CCD detector \\
Space group & P212121 \\
a, b, c $(\AA)$ & $37.48,67.22,102.41$ \\
Resolution range $(\AA)$ & $50.00-1.79$ \\
Wavelength $(\AA)$ & 1.54 \\
Average redundancy & $4.0(2.6)$ \\
Unique reflections & $25021(3013)$ \\
Completeness $(\%)$ & $99.1(97.7)$ \\
R merge $(\%)$ & $6.1(34.2)$ \\
Average $/ / \sigma(\mathrm{I})$ & $27.4(4.0)$ \\
Asymmetric unit & Two molecules \\
R/R-free & $0.178 / 0.233$ \\
No. of atoms & 2353 \\
No. of residues & 259 \\
Nof water molecules & 297 \\
Mean B value $\left(\AA^{2}\right)$ & 24.745 \\
R.m.s. bonds $(\AA)$ & 0.018 \\
R.m.s. angles $\left({ }^{\circ}\right)$ & 1.841 \\
\hline
\end{tabular}


Table 1. Cont.

\begin{tabular}{cc}
\hline Protein & TmArgBP D1 $^{\text {F57W }}$ \\
\hline MolProbity statistics & \\
\hline MolProbity score & 1.47 \\
Clashscore, all atoms: & 3.86 \\
Ramachandran Allowed & $257 / 257$ \\
Ramachandran Favored & $246 / 257$ \\
Ramachandran Outliers & $0 / 257$ \\
Favored rotamers & $212 / 221$ \\
Poor rotamers & $2 / 221$ \\
C $\beta$ deviation outliers & $2 / 237$ \\
Bad bonds & $2 / 2074$ \\
Bad angles & $1 / 2795$ \\
\hline
\end{tabular}

A comparative analysis of the global structure of $\mathrm{D} 1^{\mathrm{F57W}}$ (Figure 7A) with previous structural characterizations of D1 in different binding states or embodied in the native protein indicates that the mutation does not produce major rearrangements (Table S2). The inspection of the binding pocket (Figure 7 and Figure S8) indicates that the arginine is anchored by a remarkable number of residues that establish a variety of different interactions with the ligand [21]. A remarkable contribution to the recognition of arg is provided by the mutated residue W57 (Figure 7, Figure S8A,C). The values of the side chain dihedral angles $\left(\chi^{1} ; \chi^{2}\right)$ that the residue adopts in the crystallographic structure are $\left(-178.16^{\circ},-92.18^{\circ}\right)$ and $\left(174.49^{\circ},-108.34^{\circ}\right)$ for the two molecules present in the asymmetric unit. This observation indicates that W57 assumes the rotameric state predicted by the computational analysis reported above. Notably, the accessible surface area of this residue decreases from $104.9 \AA^{2}$, in the absence of the ligand, to $31.6 \AA^{2}$. The drastic change in the local environment of the tryptophan explains the variation of the fluorescence signal associated with the arginine binding. It is worth mentioning that, in addition to the guanidinium moiety, W57 interacts with several other atoms (Table S3). This observation indicates that almost the entire arg aminoacid contributes to the burying of tryptophan side chain and explains why the binding of the sole guanidinium moiety produces limited effects on the fluorescence emission (Figure 6D).

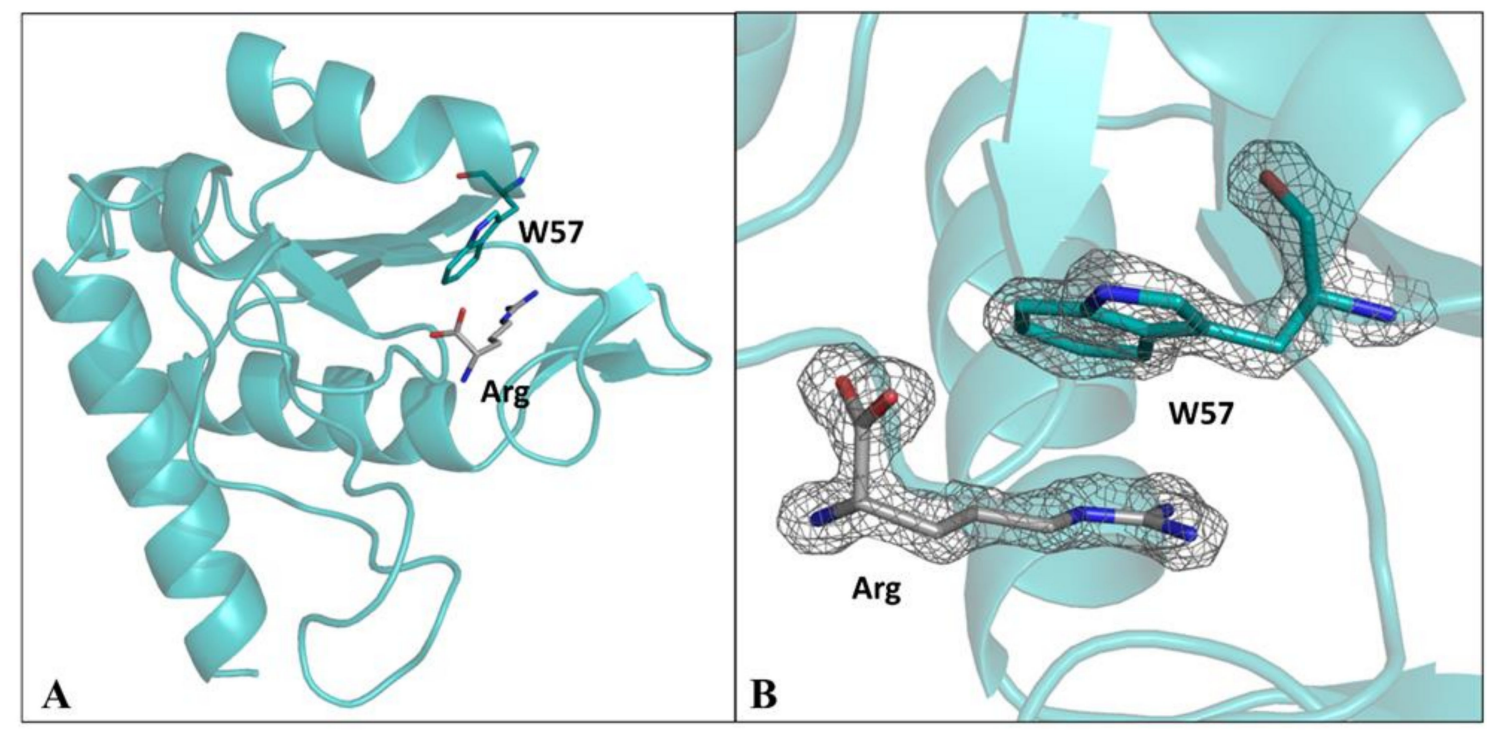

Figure 7. Crystal structure of the arginine-bound D ${ }^{\mathrm{F} 57 \mathrm{~W}}$ from TmArgBP. The arginine ligand and W57 residue are shown as sticks (A). $|2 \mathrm{Fo}-\mathrm{Fc}|$ electron density map, contoured at $2.0 \sigma$, of the arginine ligand and of the mutated residue W57 (B). 


\section{Discussion}

The detection of arginine is an issue whose importance extends over different scientific areas. In biomedical sciences, the role of this aminoacid goes well beyond its contribution to the building of proteins. Indeed, arginine dysregulations underlie several pathological states [24]. The absence in melanoma and hepatocellular carcinoma of the enzyme argininosuccinate synthase makes the production of arginine unfeasible [25]. Low levels of arginine are found in several cancers, including leukemia and breast cancer, as well as in other clinical states, such as asthma, arthritis and psoriasis [26-29]. On the other hand, the quantification of arginine in food is an important quality control step, as products of arginine degradation may lead to the formation of carcinogens. In this scenario, it is not surprising that a repertoire of different methods for arginine detection have been developed [30,31]. As insightfully reviewed by Verma et al. [24], these methods, which have been developed in a variety of instrumental conditions, exploit enzymatic essays as well as whole cell and tissue-based tools. Although natural proteins often lack some key requisites, such as specificity and stability, to be used as biosensors there is a growing interest in developing protein-based biosensors. Substrate-binding proteins represent promising candidates to be used as scaffold for biosensors, although substantial engineering may be required. This approach has been applied with some success also for the arginine detection $[16,20,32,33]$. Here, we exploited the extraordinary stability of TmArgBP to generate novel and simplified scaffolds whose tryptophan fluorescence depends on their arginine binding state. In particular, the possibility to truncate and/or dissect the protein into fragments that are endowed with a remarkable stability against temperature $\left(>90^{\circ} \mathrm{C}\right)$, chemical denaturants, and pressure (10 gigabar) [12,17] prompted us to make Trp fluorescent variants that were sensitive to arginine upload. In this framework, we developed two different scaffolds corresponding to the truncated monomeric form of the protein devoid of its C-terminal swapping helix and to the individual D1 domain. In both cases, the replacement of a Phe residue of the binding pocket with a Trp residue was sufficient to detect a clear variation of the Trp fluorescence upon ligand biding while retaining the remarkable stability of the parent protein. It is also worth mentioning that the removal of the $C$-terminal end from these constructs also eliminates the only other Trp residue present in the TmArgBP sequence. Despite the extreme simplicity of $\mathrm{D} 1^{\mathrm{F} 7 \mathrm{~W}}$, the individual domain retains a good affinity for arginine $\left(\mathrm{k}_{\mathrm{D}}\right.$ of 3 $\mu \mathrm{M})$. Surprisingly, the larger TmArgBP $20-233 \_F 76 \mathrm{~W}$, in which both D1 and D2 domains cooperate to the anchoring of the arginine, presents a reduced affinity $\left(\mathrm{k}_{\mathrm{D}}\right.$ of $\left.\sim 100 \mu \mathrm{M}\right)$. Interestingly, the two scaffolds have been designed to exhibit a completely different behavior in relation to the arginine release. Indeed, while TmArgBP $20-233$ F76W , in line with the ligand burying shown by SBP, binds the ligand in an almost irreversible way, $\mathrm{D} 1^{\mathrm{F} 57 \mathrm{~W}}$ releases the ligand with a simple washing. It is also worth mentioning that the binding of the guanidinium ion does not produce fluorescence variations. This finding indirectly suggests that small contaminants deriving from arginine degradation should not interfere with the aminoacid quantification. Altogether, we here report the development of two TmArgBP-based different scaffolds for arginine sensing characterized by different sizes and properties. In a more general context, our data indicate that SBP isolated from thermophilic organisms may be effectively engineered to generate miniaturized variants of these proteins that can be used as protein-based biosensors.

\section{Materials and Methods}

\subsection{Notations}

Wild-type TmArgBP is a dimeric protein made of 247 residues (UniProtKB - Q9WZ62). Its $N$-terminal end (residues 1-19) is the signal peptide responsible for the periplasmic localization of the protein. Since this region is highly hydrophobic, it is typically removed in the recombinant forms of the protein. Therefore, the monomeric form of the protein generated through the truncation of the C-terminal helix corresponds to the residues 20-233 (TmArgBP ${ }^{20-233}$ ). In the numbering scheme of the wild-type and of the truncated protein the Phe $\rightarrow \operatorname{Trp}$ mutation characterized here corresponds to residue 76 . Since the D1 domain of the protein is made of fragments that are non-consecutive in 
TmArgBP sequence (residues 20-114 and 207-233), which were artificially connected by a GGGGSG flexible linker, its numbering cannot follow that of the wild-type protein. Therefore, in line with previous studies, the numbering of D1 starts with the residue 1 that corresponds to the residue 20 of the wild-type protein. Consequently, the Phe $\rightarrow \operatorname{Trp}$ mutation here characterized corresponds to residue 57 in the D1 framework.

\subsection{Molecular Dynamics: Models and Protocol}

Fully atomistic Molecular Dynamics (MD) simulations were performed on the arginine bound form of TmArgBP $20-233 \_F 76 \mathrm{~W}$. Atomic coordinates of TmArgBP ${ }^{20-233}$ were extracted from the PDB entry 6GGV. Residue Phe76 was mutated to Trp using COOT (Crystallographic Object-Oriented Toolkit) [34]. The MD simulation was carried out using the GROMACS (GROningen MAchine for Chemical Simulations) software package (version 2019.6) and Amber99SB as force field [35]. The protein model was immersed in a triclinic box $\left(6.042 \times 7.806 \times 5.755 \mathrm{~nm}^{3}\right)$ and solvated with 7637 water molecules of the TIP3P (transferable intermolecular potential with 3 points) model. A minimum distance of $10 \AA$ between the protein and the box was imposed. The system was neutralized adding five sodium counterions. The particle-mesh Ewald (PME) method with a grid spacing of $1.6 \AA$ was used for the electrostatic interactions, whereas a $10 \AA$ cut-off was applied for the Lennard-Jones (LJ) interactions. Bond lengths were constrained with the LINCS (LINear Constraint Solver) algorithm [36]. The system was first energy minimized by means of steepest descent and then equilibrated in two steps: in the first phase, the temperature of the systems was stabilized at $300 \mathrm{~K}$ for $500 \mathrm{ps}$ (NVT); equilibration of pressure at $1 \mathrm{~atm}$ was then conducted for other $500 \mathrm{ps}(\mathrm{NpT})$. Temperature and pressure control were achieved using the Velocity Rescaling and Parrinello-Rahman algorithms, respectively [37]. We carried out a production run of $150 \mathrm{~ns}$ using a time step of $2 \mathrm{fs}$. The convergence of the simulation was checked by calculating the root mean square inner product (RMSIP) between the two halves of the equilibrated trajectory. To this aim, the motions of the protein $\mathrm{C}^{\alpha}$ atoms along the first 10 eigenvectors were considered $[38,39]$. In detail, the last $130 \mathrm{~ns}$ of trajectory was divided in two halves $(20-85 \mathrm{~ns}$ and $85-150 \mathrm{~ns})$. The high value of the RMSIP (0.77) between these two trajectory portions indicates that a satisfactory level of convergence is obtained. Structural analyses of trajectory frames were carried out with GROMACS routines and the VMD (Visual Molecular Dynamics) molecular visualization program [40].

\subsection{Cloning, Expression and Purification of the Mutants}

The Phe $\rightarrow$ Trp replacement in the truncated TmArgBP20-233 $\left(\operatorname{TmArgBP}{ }^{20-233-F 76 W}\right)$ and in the D1 $\left(\mathrm{D} 1^{\mathrm{F} 57 \mathrm{~W}}\right)$ was obtained using the QuickChange mutagenesis kit (Agilent) (Figure S1).

The following mutagenic primers were used in this study: F76Fw-5' GAAGATCGTCGA TATGACCTGGGACGGACTCATTCCGAGCC-3' and F76WRv-5' GGCTCGGAATGAGTCCGTC CCAGGTCATATCGACGATCTTC-3'. The two mutants were expressed using Escherichia coli (E. coli) BL21(DE3) or Rosetta 2 (DE3) cells. Induction was performed at $22{ }^{\circ} \mathrm{C}$ for $16 \mathrm{~h}$ by the addition of $0.8-0.5 \mathrm{mM}$ Isopropyl $\beta$-d-1-thiogalactopyranoside(IPTG). Cells were then harvested and the soluble extract was obtained by sonicating cell pellets in $20 \mathrm{~mL}$ of lysis buffer. The purification of $\operatorname{TmArgBP}{ }^{20-233 \_F 76 W}$ and $1^{\mathrm{F} 57 \mathrm{~W}}$ was conducted in a fashion similar to that previously reported $[9,11]$. To further purify soluble proteins from aggregated forms and/or other contaminants, fractions containing TmArgBP ${ }^{20-233 \_F 76 W}$ and D1 ${ }^{\mathrm{F} 57 \mathrm{~W}}$ were loaded on Superdex S75 10/30 pre-equilibrated in a buffer containing $50 \mathrm{mM}$ Tris $\mathrm{HCl}$ and $150 \mathrm{mM} \mathrm{NaCl}$ (pH 8.0). The ligand-free form of the two proteins was obtained using two different protocols. In the case of TmArgBP $20-233-F 76 \mathrm{~W}$, the arginine was removed by dialysis using strong denaturing conditions in the presence of $4 \mathrm{M} \mathrm{GuHCl}$ [10], whereas, for $\mathrm{D}^{\mathrm{F} 57 \mathrm{~W}}$, a prolonged dialysis in Tris/ $\mathrm{NaCl}$ buffer was sufficient. 


\subsection{Circular Dichroism (CD)}

CD spectra were registered with a J-810 spectropolarimeter equipped with a Peltier temperature control system (model PTC-423-S, Jasco Europe, Cremella (LC), Italy). Far-ultraviolet measurements $(198-260 \mathrm{~nm})$ were performed at $20^{\circ} \mathrm{C}$ using a $0.1 \mathrm{~cm}$ optical path length cell. The proteins were dissolved in phosphate buffer saline (PBS) buffer ( $\mathrm{pH} 7.4)$ and tested at $0.2 \mathrm{mg} / \mathrm{mL}$ concentration. CD spectra were recorded with a time constant of $4 \mathrm{~s}$, a $2 \mathrm{~nm}$ bandwidth, and a scan rate of $10 \mathrm{~nm} \mathrm{~min}{ }^{-1}$. The reported spectra were obtained after averaging the signal over at least three scans. The baseline was corrected by subtracting the complete buffer spectrum. Thermal denaturation curves were recorded over the $20^{\circ} \mathrm{C}-100^{\circ} \mathrm{C}$ temperature range monitoring the $\mathrm{CD}$ signal at $222 \mathrm{~nm}$. The curve was registered either by simply dissolving the protein in the PBS buffer or by adding $4.0 \mathrm{M} \mathrm{GuHCl}$ to this solution.

\subsection{Tryptofan Fluorescence Spectroscopy (TFS)}

All tryptophan fluorescence spectroscopy experiments were performed using CARY Eclipse Fluorescence Spectrophotometer (Varian Inc. Santa Clara, CA, USA) and a $100 \mu$ l quartz cuvette. The excitation wavelength was fixed to $263 \mathrm{~nm}$ and emission spectra were collected between 300 and $500 \mathrm{~nm}$ with a slit width of $5 \mathrm{~nm}$. The temperature was kept constant at $25^{\circ} \mathrm{C}$. To measure arginine interactions, recombinant proteins were used at a concentration of $45 \mu \mathrm{M}$ in Tris buffer ( $\mathrm{pH}$ 8.0) and the fluorescence spectra were reordered with increasing concentrations of ligand from 0 to $730 \mu \mathrm{M}$. Control buffer titrations to protein solutions were performed in parallel for background determination in each experiment. Data derived from three independent experiments were analyzed using nonlinear regression with GraphPad Prism 6 by applying the binding model "One Site- Total". To test the reversibility of the ligand binding, after the analysis, the protein-ligand mixture was rinsed with Tris buffer three/four times using a centrifugal device (Amicon Ultra centrifugal filter, Millipore). After the washing, the arginine binding was tested again by TFS.

\subsection{Protein Crystallization, Data Collection and Structure Refinement}

Crystallization experiments were performed at $293 \mathrm{~K}$ on a solution containing the mutated D1 domain by using the hanging-drop vapor diffusion method. Crystals were obtained using a protein concentration of $6 \mathrm{mg} \mathrm{mL}^{-1}$ and a medium containing $0.1 \mathrm{M}$ Sodium acetate trihydrate $\mathrm{pH} \mathrm{4.5,25 \%}$ $w / v$ Polyethylene glycol 3350. High-resolution diffraction data were collected in house using $\mathrm{Cu} \mathrm{K} \alpha$ X-ray radiation (1.54 ^̊) from a Rigaku Micromax 007 HF generator equipped with a Saturn944 CCD detector at $100 \mathrm{~K}$. The data were collected by flash-cooling in the supercooled $\mathrm{N}_{2}$ gas produced by an Oxford Cryo-system after the addition of $20 \%(v / v)$ ethylene glycol to the harvesting solution. Data were processed and scaled using the HKL2000 program package. The crystals, which contain two molecules per asymmetric unit, belong to the P 212121 space group and diffracted at $1.79 \AA$. Data collection statistics are reported in Table 1.

The structure of the protein was solved by molecular replacement using the program Phaser [41] and the structure of the arginine-bound form of D1 (PDB code 6GPC) as a starting model. A reliable model was then obtained by the automatic modeling performed using ARP/wARP [42]. The crystallographic refinement of the structure has been performed with REFMAC (REFinement of MACromolecular structures) [43]. Since the early stage of the refinement, a clear electron density corresponding to the indole group of the Trp side chain at position 57 was evident in both chains. The electron density is generally of good quality along the entire polypeptide chains. Water molecules were incorporated into the structure in several rounds of refinements. The final model presents R-factor and R-free values of 0.178 and 0.233 , respectively. The stereochemistry of the final model was checked by using standard validation protocols such as PDB validator (https://validate-rcsb-2.wwpdb.org/) and Molprobity [44] and innovative approaches that are based on the evaluation of the conformation-dependent variability of the peptide geometry (http://study.ibb.cnr.it/quiproqua/) [45-47]. The analysis of the final model carried out with the 
Molprobity server indicates that it is endowed with a good stereochemistry (Table 1). Indeed, the $\varphi$ and $\psi$ values of all residues fall in allowed regions of the Ramachandran plot with the vast majority adopting favored conformations (95.7\%). Finally, to evaluate the accuracy of bond and dihedral angles of the final model, we checked the variability of the backbone valence angles and of the peptide planarity as a function of the local conformation. As shown in Table S1 and Figure S6, the variability of these parameters follows the trends detected in highly accurate protein structures [45-47]. The atomic coordinates of $\mathrm{D}^{\mathrm{F} 57 \mathrm{~W}}$ have been deposited in the PDB with the identification code 7A99.

Supplementary Materials: The following are available online at http://www.mdpi.com/1422-0067/21/20/7503/s1, Figure S1: Amino acid sequence of the wild-type TmArgBP and of the two protein variants TmArgBP $20-233 \_F 76 \mathrm{~W}$; Figure S2: Cartoon representation of the crystal structure of the arginine-bound form of TmArgBP20-233; Figure S3: Modelling of the Phe76Trp mutation in the arginine-bound form of TmArgBP20-233; Figure S4: Stability of TmArgBP20-233_F76W in the MD simulation; Figure S5: MD simulation of TmArgBP20-233_F76W; Figure S6: Hydrophobic interactions in the MD simulation of TmArgBP20-233_F76W; Figure S7: Validation of the variability of geometrical parameters of the protein backbone in the crystallographic structure of TmArgBP D1 ${ }^{\text {F57W }}$; Figure S8: Network of the interactions established by Arg with the protein residues in the crystal structure of D1 ${ }^{\mathrm{F} 57 \mathrm{~W}}$; Table S1: Results of the regression analysis of the variability of several bond angles. Table S2: Structural comparison of TmArgBP D1 ${ }^{\mathrm{F} 57 \mathrm{~W}}$, D1 and TmArgBP ${ }^{20-233}$; Table S3: Atoms of the arginine bound to TmArgBP D1 $1^{\mathrm{F} 57 \mathrm{~W}}$ that are closer than $4.0 \AA$ to atoms of W57 side chain.

Author Contributions: Conceptualization, All authors; methodology, G.S, A.R.; investigation, G.S., A.R., N.B.; data curation, All authors; writing-original draft preparation, L.V.; writing-review and editing, All authors; supervision, G.S., A.R. All authors have read and agreed to the published version of the manuscript.

Funding: This work was supported by "Progetti di Ricerca Corrente" funded by the Italian Ministry of Health covering the publication fee.

Acknowledgments: The authors thank Luca De Luca and Maurizio Amendola for technical support and Mirjana Paladino for her contribution in the early stage of the work. CINECA Supercomputing (ISCRA C project code HP10CFJL92 - ArgSens) is acknowledged for computational support.

Conflicts of Interest: The authors declare no conflict of interest.

\section{Abbreviations}

$\begin{array}{ll}\text { TmArgBP } & \text { Arginine-binding protein from Thermotoga maritima } \\ \text { TmArgBP20-233 } & \text { Region encompassing residues 20-233 of TmArgBP } \\ \text { D1 } & \text { Domain 1 of TmArgBP (residues 20-114 and 207-233 linked by a GGGGSG segment) } \\ \text { SBP } & \text { Substrate-binding proteins } \\ \text { MD } & \text { Molecular dynamics } \\ \text { GuHCl } & \text { Guanidinium chloride } \\ \text { PDB } & \text { Protein Data Bank } \\ \text { RMSD } & \text { Root-Mean-Square Deviation }\end{array}$

\section{References}

1. Tapiero, H.; Mathé, G.; Couvreur, P.; Tew, K.D.I. Arginine. Biomed. Pharmacother. 2002, 56, 439-445. [CrossRef]

2. Andrew, P.J.; Mayer, B. Enzymatic function of nitric oxide synthases. Cardiovasc. Res. 1999, 43, 521-531. [CrossRef]

3. Wheatley, D.N.; Scott, L.; Lamb, J.; Smith, S. Single amino acid (arginine) restriction: Growth and death of cultured HeLa and human diploid fibroblasts. Cell. Physiol. Biochem. 2000, 10, 37-55. [CrossRef] [PubMed]

4. Bednarz-Misa, I.; Fortuna, P.; Fleszar, M.G.; Lewandowski, Ł.; Diakowska, D.; Rosińczuk, J.; Krzystek-Korpacka, M. Esophageal Squamous Cell Carcinoma Is Accompanied by Local and Systemic Changes in L-arginine/NO Pathway. Int. J. Mol. Sci. 2020, 21. [CrossRef]

5. Xiong, L.; Teng, J.L.; Botelho, M.G.; Lo, R.C.; Lau, S.K.; Woo, P.C. Arginine Metabolism in Bacterial Pathogenesis and Cancer Therapy. Int. J. Mol. Sci. 2016, 17, 363. [CrossRef]

6. Morris, S.M. Arginine Metabolism Revisited. J. Nutr. 2016, 146, 2579S-2586S. [CrossRef] [PubMed]

7. Scaglia, F.; Lee, B. Clinical, biochemical, and molecular spectrum of hyperargininemia due to arginase I deficiency. Am. J. Med. Genet. C Semin Med. Genet. 2006, 142C, 113-120. [CrossRef] 
8. Jęśko, H.; Wilkaniec, A.; Cieślik, M.; Hilgier, W.; Gąssowska, M.; Lukiw, W.J.; Adamczyk, A. Altered Arginine Metabolism in Cells Transfected with Human Wild-Type Beta Amyloid Precursor Protein ( $\beta$ APP). Curr. Alzheimer Res. 2016, 13, 1030-1039. [CrossRef]

9. Ruggiero, A.; Dattelbaum, J.D.; Staiano, M.; Berisio, R.; D’Auria, S.; Vitagliano, L. A loose domain swapping organization confers a remarkable stability to the dimeric structure of the arginine binding protein from Thermotoga maritima. PLoS ONE 2014, 9, e96560. [CrossRef]

10. Smaldone, G.; Berisio, R.; Balasco, N.; D'Auria, S.; Vitagliano, L.; Ruggiero, A. Domain swapping dissection in Thermotoga maritima arginine binding protein: How structural flexibility may compensate destabilization. Biochim. Biophys. Acta Proteins Proteom. 2018, 1866, 952-962. [CrossRef]

11. Smaldone, G.; Balasco, N.; Vigorita, M.; Ruggiero, A.; Cozzolino, S.; Berisio, R.; Del Vecchio, P.; Graziano, G.; Vitagliano, L. Domain communication in Thermotoga maritima Arginine Binding Protein unraveled through protein dissection. Int. J. Biol. Macromol. 2018, 119, 758-769. [CrossRef] [PubMed]

12. Jaworek, M.W.; Ruggiero, A.; Graziano, G.; Winter, R.; Vitagliano, L. On the extraordinary pressure stability of the Thermotoga maritima arginine binding protein and its folded fragments-A high-pressure FTIR spectroscopy study. Phys. Chem. Chem. Phys. 2020, 22, 11244-11248. [CrossRef] [PubMed]

13. Balasco, N.; Smaldone, G.; Vigorita, M.; Del Vecchio, P.; Graziano, G.; Ruggiero, A.; Vitagliano, L. The characterization of Thermotoga maritima Arginine Binding Protein variants demonstrates that minimal local strains have an important impact on protein stability. Sci. Rep. 2019, 9, 6617. [CrossRef] [PubMed]

14. Smaldone, G.; Vigorita, M.; Ruggiero, A.; Balasco, N.; Dattelbaum, J.D.; D'Auria, S.; Del Vecchio, P.; Graziano, G.; Vitagliano, L. Proline 235 plays a key role in the regulation of the oligomeric states of Thermotoga maritima Arginine Binding Protein. Biochim. Biophys. Acta 2016, 1864, 814-824. [CrossRef]

15. Smaldone, G.; Ruggiero, A.; Balasco, N.; Abuhammad, A.; Autiero, I.; Caruso, D.; Esposito, D.; Ferraro, G.; Gelardi, E.L.M.; Moreira, M.; et al. The non-swapped monomeric structure of the arginine-binding protein from Thermotoga maritima. Acta Crystallogr. F Struct. Biol. Commun. 2019, 75, 707-713. [CrossRef] [PubMed]

16. Deacon, L.J.; Billones, H.; Galyean, A.A.; Donaldson, T.; Pennacchio, A.; Iozzino, L.; D'Auria, S.; Dattelbaum, J.D. Tryptophan-scanning mutagenesis of the ligand binding pocket in Thermotoga maritima arginine-binding protein. Biochimie 2014, 99, 208-214. [CrossRef]

17. Cozzolino, S.; Balasco, N.; Vigorita, M.; Ruggiero, A.; Smaldone, G.; Del Vecchio, P.; Vitagliano, L.; Graziano, G. Guanidinium binding to proteins: The intriguing effects on the D1 and D2 domains of Thermotoga maritima Arginine Binding Protein and a comprehensive analysis of the Protein Data Bank. Int. J. Biol. Macromol. 2020, 163, 375-385. [CrossRef]

18. Scirè, A.; Marabotti, A.; Staiano, M.; Iozzino, L.; Luchansky, M.S.; Der, B.S.; Dattelbaum, J.D.; Tanfani, F.; D'Auria, S. Amino acid transport in thermophiles: Characterization of an arginine-binding protein in Thermotoga maritima. 2. Molecular organization and structural stability. Mol. Biosyst. 2010, 6, 687-698. [CrossRef]

19. Luchansky, M.S.; Der, B.S.; D’Auria, S.; Pocsfalvi, G.; Iozzino, L.; Marasco, D.; Dattelbaum, J.D. Amino acid transport in thermophiles: Characterization of an arginine-binding protein in Thermotoga maritima. Mol. Biosyst. 2010, 6, 142-151. [CrossRef]

20. Donaldson, T.; Iozzino, L.; Deacon, L.J.; Billones, H.; Ausili, A.; D’Auria, S.; Dattelbaum, J.D. Engineering a switch-based biosensor for arginine using a Thermotoga maritima periplasmic binding protein. Anal. Biochem. 2017, 525, 60-66. [CrossRef]

21. Laskowski, R.A.; Swindells, M.B. LigPlot+: Multiple Ligand-Protein Interaction Diagrams for Drug Discovery. J. Chem. Inf. Model. 2011, 51, 2778-2786. [CrossRef] [PubMed]

22. Lovell, S.C.; Word, J.M.; Richardson, J.S.; Richardson, D.C. The penultimate rotamer library. Proteins 2000, 40, 389-408. [CrossRef]

23. Munishkina, L.A.; Fink, A.L. Fluorescence as a method to reveal structures and membrane-interactions of amyloidogenic proteins. Biochim. Biophys. Acta 2007, 1768, 1862-1885. [CrossRef] [PubMed]

24. Verma, N.; Singh, A.K.; Singh, M. L-arginine biosensors: A comprehensive review. Biochem. Biophys. Rep. 2017, 12, 228-239. [CrossRef] [PubMed] 
25. Ensor, C.M.; Holtsberg, F.W.; Bomalaski, J.S.; Clark, M.A. Pegylated arginine deiminase (ADI-SS PEG20,000 $\mathrm{mw}$ ) inhibits human melanomas and hepatocellular carcinomas in vitro and in vivo. Cancer Res. 2002, 62, 5443-5450.

26. Wheatley, D.N.; Philip, R.; Campbell, E. Arginine deprivation and tumour cell death: Arginase and its inhibition. Mol. Cell Biochem. 2003, 244, 177-185. [CrossRef]

27. Wheatley, D.N.; Campbell, E. Arginine deprivation, growth inhibition and tumour cell death: 3 . Deficient utilisation of citrulline by malignant cells. Br. J. Cancer 2003, 89, 573-576. [CrossRef]

28. Bruch-Gerharz, D.; Schnorr, O.; Suschek, C.; Beck, K.F.; Pfeilschifter, J.; Ruzicka, T.; Kolb-Bachofen, V. Arginase 1 overexpression in psoriasis: Limitation of inducible nitric oxide synthase activity as a molecular mechanism for keratinocyte hyperproliferation. Am. J. Pathol. 2003, 162, 203-211. [CrossRef]

29. Meurs, H.; Maarsingh, H.; Zaagsma, J. Arginase and asthma: Novel insights into nitric oxide homeostasis and airway hyperresponsiveness. Trends Pharmacol. Sci. 2003, 24, 450-455. [CrossRef]

30. Ginésy, M.; Enman, J.; Rusanova-Naydenova, D.; Rova, U. Simultaneous Quantification of L-arginine and Monosaccharides during Fermentation: An Advanced Chromatography Approach. Molecules 2019, 24. [CrossRef]

31. Tam, S.Y.; Chung, S.F.; Chen, Y.W.; So, Y.H.; So, P.K.; Cheong, W.L.; Wong, K.Y.; Leung, Y.C. Design of a structure-based fluorescent biosensor from bioengineered arginine deiminase for rapid determination of L-arginine. Int. J. Biol. Macromol. 2020. [CrossRef] [PubMed]

32. Ausili, A.; Staiano, M.; Dattelbaum, J.; Varriale, A.; Capo, A.; D'Auria, S. Periplasmic Binding Proteins in Thermophiles: Characterization and Potential Application of an Arginine-Binding Protein from Thermotoga maritima: A Brief Thermo-Story. Life (Basel) 2013, 3, 149-160. [CrossRef] [PubMed]

33. Whitfield, J.H.; Zhang, W.H.; Herde, M.K.; Clifton, B.E.; Radziejewski, J.; Janovjak, H.; Henneberger, C.; Jackson, C.J. Construction of a robust and sensitive arginine biosensor through ancestral protein reconstruction. Protein Sci. 2015, 24, 1412-1422. [CrossRef] [PubMed]

34. Emsley, P.; Lohkamp, B.; Scott, W.G.; Cowtan, K. Features and development of Coot. Acta Crystallogr. D Biol. Crystallogr. 2010, 66, 486-501. [CrossRef] [PubMed]

35. Van Der Spoel, D.; Lindahl, E.; Hess, B.; Groenhof, G.; Mark, A.E.; Berendsen, H.J. GROMACS: Fast, flexible, and free. J. Comput Chem 2005, 26, 1701-1718. [CrossRef]

36. Hess, B. P-LINCS: A Parallel Linear Constraint Solver for Molecular Simulation. J. Chem. Theory Comput. 2008, 4, 116-122. [CrossRef]

37. Bussi, G.; Donadio, D.; Parrinello, M. Canonical sampling through velocity rescaling. J. Chem. Phys. 2007, 126, 014101. [CrossRef]

38. Amadei, A.; Ceruso, M.A.; Di Nola, A. On the convergence of the conformational coordinates basis set obtained by the essential dynamics analysis of proteins' molecular dynamics simulations. Proteins 1999, 36, 419-424. [CrossRef]

39. Merlino, A.; Vitagliano, L.; Ceruso, M.A.; Mazzarella, L. Subtle functional collective motions in pancreatic-like ribonucleases: From ribonuclease A to angiogenin. Proteins 2003, 53, 101-110. [CrossRef]

40. Humphrey, W.; Dalke, A.; Schulten, K. VMD: Visual molecular dynamics. J. Mol. Graph. 1996, 14, 33-38. [CrossRef]

41. McCoy, A.J.; Grosse-Kunstleve, R.W.; Adams, P.D.; Winn, M.D.; Storoni, L.C.; Read, R.J. Phaser crystallographic software. J. Appl. Crystallogr. 2007, 40, 658-674. [CrossRef] [PubMed]

42. Morris, R.J.; Perrakis, A.; Lamzin, V.S. ARP/wARP and automatic interpretation of protein electron density maps. Methods Enzymol. 2003, 374, 229-244. [CrossRef] [PubMed]

43. Murshudov, G.N.; Vagin, A.A.; Dodson, E.J. Refinement of macromolecular structures by the maximum-likelihood method. Acta Crystallogr. D Biol. Crystallogr. 1997, 53, 240-255. [CrossRef] [PubMed]

44. Chen, V.B.; Arendall, W.B.; Headd, J.J.; Keedy, D.A.; Immormino, R.M.; Kapral, G.J.; Murray, L.W.; Richardson, J.S.; Richardson, D.C. MolProbity: All-atom structure validation for macromolecular crystallography. Acta Crystallogr. D Biol. Crystallogr. 2010, 66, 12-21. [CrossRef]

45. Improta, R.; Vitagliano, L.; Esposito, L. Peptide bond distortions from planarity: New insights from quantum mechanical calculations and peptide/protein crystal structures. PLoS ONE 2011, 6, e24533. [CrossRef] 
46. Balasco, N.; Esposito, L.; Vitagliano, L. Factors affecting the amplitude of the $\tau$ angle in proteins: A revisitation. Acta Crystallogr. D Struct. Biol. 2017, 73, 618-625. [CrossRef]

47. Balasco, N.; Esposito, L.; Thind, A.S.; Guarracino, M.R.; Vitagliano, L. Dissection of Factors Affecting the Variability of the Peptide Bond Geometry and Planarity. Biomed. Res. Int. 2017, 2017, 2617629. [CrossRef] 